\title{
Decreasing fall risk in spinocerebellar ataxia
}

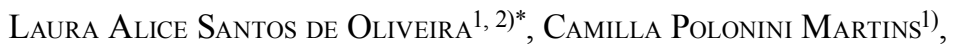 \\ Carlos Henrique Ramos Horsczaruk ${ }^{1)}$, Débora Cristina Lima da Silva ${ }^{1)}$, \\ José Vicente Pereira Martins ${ }^{3)}$, Luiz Felipe Rocha Vasconcelos ${ }^{3)}$, \\ Erika de Carvalho Rodrigues ${ }^{1,2)}$ \\ 1) Post-graduation Program in Rehabilitation Sciences, Augusto Motta University Center: Praça das \\ Nações, 34, $3^{\circ}$ andar, Rio de Janeiro 21041-020, Brazil \\ 2) Federal Institute of Rio de Janeiro, Brazil \\ 3) Federal University of Rio de Janeiro, Deolindo Couto Neurology Institute, Brazil
}

\begin{abstract}
Purpose] Spinocerebellar ataxia consists of a group of autosomal dominant disorders that cause progressive degeneration, mainly in the cerebellum and its connections. Falls, which are a significant concern of this condition, reduce patients' mobility, deteriorate their health and have physical and social consequences. The aim of this study was to test the effectiveness of a modified protocol for improving balance and diminishing the fall risk of spinocerebellar ataxia patients exclusively. [Subjects and Methods] Exercises aiming to improve static and dynamic balance, whole body movements, measures to prevent falls and falling strategies were performed twice per week for four weeks by 11 spinocerebellar ataxia patients. Balance was evaluated using the Berg Balance Scale. [Results] The results show that there was a significant increase in Berg Balance Scale scores after the interventions (Wilcoxon $\mathrm{p}=0.0034$ ). [Conclusion] This study demonstrated that the modified protocol is effective at reducing the fall risk of spinocerebellar ataxia patients. This protocol may be a useful option for appropriately coping with falls caused by spinocerebellar ataxia.

Key words: SCA, Balance, Physical therapy
\end{abstract}

(This article was submitted Oct. 27, 2014, and was accepted Dec. 16, 2014)

\section{INTRODUCTION}

Spinocerebellar ataxia (SCA) consists of a group of autosomal dominant disorders that cause progressive degeneration in the cerebellum and its connections, gait and limb ataxic movements ${ }^{1)}$. SCA is often accompanied by degeneration at other sites in the nervous system that produce non-cerebellar signs such as pyramidal and extrapyramidal deficits $^{2}$.

One of the challenges of living with SCA is balance impairments, which are a key risk factor of falls ${ }^{3)}$. In one study, the number of falls in 228 SCA patients was determined during a one year period preceding a survey ${ }^{4}$. Notably, 73.6\% of these patients reported at least one fall during this period, and a high rate of fall-related injuries was also reported (74\%). Later, a prospective study) with 113 SCA patients that recorded their falls during one year in a diary showed that $84.1 \%$ of the patients reported at least one fall. This high fall risk can reduce mobility, deteriorate general health, and has physical and social consequences ${ }^{6}$. Thus, the develop-

*Corresponding author. Laura Alice Santos de Oliveira (E-mail: lauraoliveira.ft@gmail.com)

C2015 The Society of Physical Therapy Science. Published by IPEC Inc. This is an open-access article distributed under the terms of the Creative Commons Attribution Non-Commercial No Derivatives (by-ncnd) License $<$ http://creativecommons.org/licenses/by-nc-nd/3.0/>. ment of strategies to improve balance and decrease the fall risk is critical for SCA patients.

To date, there is no pharmacological treatment which can minimize the motor symptoms related to SCA. Physiotherapy approaches are one of the few treatments that can address this condition ${ }^{7}$.

In a previous study, an intervention to improve coordination and balance was conducted with 15 patients with hereditary cerebellar ataxia ${ }^{7}$. This protocol was performed for one hour, three times a week, for four weeks. It reduced ataxia symptoms and improved motor performance and balance in static/dynamic tasks. After the four-week period, all patients were instructed to continue exercising at home for one hour every day. Some of the benefits produced by this protocol lasted for one year ${ }^{8}$. Unfortunately, among the enrolled patients, only 3 exhibited SCA. Thus, the effectiveness of this protocol cannot be directly determined for SCA patients because their characteristics include more infrequent non-ataxic signs than cerebellar ataxic patients with other etiologies.

The aim of the present study was to test the effectiveness of a modified version of this protocol ${ }^{7)}$ for improving balance and diminishing the fall risk SCA patients, exclusively. The protocol modifications reduced the number of sessions per week as well as the duration to improve the adherence of SCA outpatients to the ambulatory rehabilitation. 


\section{SUBJECTS AND METHODS}

Twelve SCA patients (7 females), aged between 28-59 years, participated in this study (Table 1). All patients provided their informed written consent. This study was approved by the local ethics committee (UNISUAM) and was conducted conform to the ethical principles of the Declaration of Helsinki.

All patients were diagnosed with SCA by a neurologist and were able to walk 10 meters. The exclusion criteria consisted of vertigo, hypertension, postural hypotension, heart disease, epilepsy or orthopedic problems.

The Brazilian version of the Berg Balance Scale (BBS), which includes 14 static and dynamic balance activities, was used to evaluate the balance and fall risk of patients ${ }^{9}$. For each item, a score ranging from $0-4$, with " 0 " indicating the lowest and " 4 " representing the highest level of function, was used to quantify the balance performance of the patients. The maximum possible BBS score is 56 . Declines in BBS scores are associated with a nonlinear increase in the fall risk ${ }^{10)}$.

The original protocol ${ }^{7)}$ included four groups of exercises: static and dynamic balance; whole body movements; measures to prevent falling and falling strategies; and movements to treat or prevent contracture. In this study, we excluded the last group of exercises because they are not directly related to falls. This modification decreased the duration of each session. The number of sessions per week was also reduced to two. The patients were encouraged to perform all exercises ten times with no external support. In some cases, the patients progressed from minimal support to no support at all.

The Wilcoxon matched-pairs signed-rank test was used to compare the BBS scores recorded before and after the intervention. A p value less than 0.05 was considered statistically significant.

\section{RESULTS}

No participants reported discomfort or suffered a fall during the protocol. One patient discontinued the treatment and was excluded from the analysis. The median age of SCA onset of the remaining 11 patients was 42 years. The majority of the patients were diagnosed with SCA3 (Table 1).

The median BBS score was 50 (Min 28, Max 54) before the intervention and $55(42,56)$ after the intervention (Table 1). The BBS score exhibited a significant increase $(p=0.0034)$ for all of the participants after the intervention.

\section{DISCUSSION}

In the present study, we utilized a modified version of a protocol used to prevent falls ${ }^{7)}$ by SCA patients. We found that there were significant improvements in static and dynamic balance as determined by the BBS score. This improvement was achieved with fewer sessions (twice a week) and a shorter duration (approximately 45 minutes) than the original protocol, which included three sessions per week of one hour each. The adaptations can increase the adherence to outpatient rehabilitation, which is restricted due to the pro-
Table 1. Individual patient characteristics

\begin{tabular}{ccccccc}
\hline \multirow{2}{*}{ Patient } & SCA Type $\begin{array}{c}\text { Age } \\
(\mathrm{yrs})\end{array}$ & Gender & $\begin{array}{c}\text { Age at } \\
\text { SCA onset }\end{array}$ & BBS & BBS \\
\hline 1 & 3 & 28 & M & 23 & 50 & 55 \\
2 & 2 & 31 & F & 20 & 50 & 52 \\
3 & 3 & 32 & M & 24 & 47 & 56 \\
4 & 2 & 45 & F & 35 & 28 & 42 \\
5 & 7 & 48 & F & 43 & 50 & 56 \\
6 & 3 & 49 & F & 35 & 35 & 45 \\
7 & 3 & 53 & F & 50 & 52 & 56 \\
8 & 3 & 53 & M & 45 & 54 & 55 \\
9 & 3 & 54 & F & 51 & 44 & 56 \\
10 & 3 & 56 & M & 42 & 54 & 56 \\
11 & 3 & 59 & M & 50 & 41 & 50 \\
\hline
\end{tabular}

BBS: Berg Balance Scale scores before and after the intervention

gressive gait and balance impairments of SCA, and because of the transportation difficulties found in less developed countries such as Brazil.

The original protocol ${ }^{7)}$ included different types of hereditary cerebellar ataxias. The present study found that there were improvements in balance in a group composed exclusively of SCA patients, indicating that the protocol may be also helpful for SCA patients.

Balance assessment is important for almost all patients receiving physical therapy, for determining appropriate therapy goals, increasing awareness of fall risk, and deciding appropriate strategies for gait ${ }^{11)}$. In the present study, the BBS score was used as an index of fall status ${ }^{10)}$. We found that the fall risk of all the participants decreased after the intervention. The fall risk is known to be high in SCA patients $^{4,5)}$. Falls can induce a vicious cycle of immobility and a fear of falling, limiting activity and social participation, thereby reducing the quality of life of patients. Therefore, the improvements reported here are relevant for SCA patients.

The key challenge in the prevention of falls is to appropriately prescribe meaningful treatments to improve this condition $^{3)}$. In the present study, we showed that interventions that improve balance and diminish the fall risk may be helpful for SCA patients.

One limitation of the present study was the absence of a control group, but it is important to remember that SCA is a progressive disorder in which balance deficits tend to deteriorate over time, making it difficult to compare results among individuals.

We conclude that a protocol focusing on improving balance and diminishing the fall risk may be advantageous for preventing falls during the rehabilitation of SCA patients.

\section{REFERENCES}

1) Shakkottai VG, Fogel BL: Clinical neurogenetics: autosomal dominant spinocerebellar ataxia. Neurol Clin, 2013, 31: 987-1007. [Medline] [CrossRef]

2) Seidel K, Siswanto S, Brunt ER, et al.: Brain pathology of spinocerebellar ataxias. Acta Neuropathol, 2012, 124: 1-21. [Medline] [CrossRef]

3) Gillespie LD, Gillespie WJ, Robertson MC, et al.: Interventions for pre- 
venting falls in elderly people. Cochrane Database Syst Rev, 2003, 2 CD000340. [Medline]

4) Fonteyn EM, Schmitz-Hübsch T, Verstappen CC, et al.: Falls in spinocerebellar ataxias: results of the EuroSCA Fall Study. Cerebellum, 2010, 9: 232-239. [Medline] [CrossRef]

5) Fonteyn EM, Schmitz-Hübsch T, Verstappen CC, et al.: Prospective analysis of falls in dominant ataxias. Eur Neurol, 2013, 69: 53-57. [Medline] [CrossRef]

6) Morton SM, Bastian AJ: Mechanisms of cerebellar gait ataxia. Cerebellum, 2007, 6: 79-86. [Medline] [CrossRef]

7) Ilg W, Synofzik M, Brötz D, et al.: Intensive coordinative training improves motor performance in degenerative cerebellar disease. Neurology, 2009, 73: 1823-1830. [Medline] [CrossRef]
8) Ilg W, Brötz D, Burkard S, et al.: Long-term effects of coordinative training in degenerative cerebellar disease. Mov Disord, 2010, 25: 2239-2246. [Medline] [CrossRef]

9) Miyamoto ST, Lombardi Junior I, Berg KO, et al.: Brazilian version of the Berg balance scale. Braz J Med Biol Res, 2004, 37: 1411-1421. [Medline] [CrossRef]

10) Shumway-Cook A, Baldwin M, Polissar NL, et al.: Predicting the probability for falls in community-dwelling older adults. Phys Ther, 1997, 77: 812-819. [Medline]

11) Bennie S, Bruner K, Dizon A, et al.: Measurements of balance: comparison of the timed "Up and Go" test and functional reach test with the Berg balance scale. J Phys Ther Sci, 2003, 15: 93-97. [CrossRef] 